\title{
Optimized Schwarz Methods for Maxwell's Equations with Non-Zero Electric Conductivity
}

Victorita Dolean ${ }^{1}$, Mohamed El Bouajaji ${ }^{2}$, Martin J. Gander ${ }^{3}$ and Stéphane Lanteri $^{2}$

1 Laboratoire J.A. Dieudonné, CNRS UMR 6621, F-06108 Nice Cedex, France

2 NACHOS project-team, INRIA Sophia Antipolis - Méditerranée research center, F-06902 Sophia Antipolis Cedex, France

3 Mathematics Section, University of Geneva, CH-1211, Geneva, Switzerland

\section{Introduction}

The first ideas for optimized Schwarz methods for Maxwell's equations came from the analysis of optimized Schwarz methods for the Helmholtz equation, see $[3,4,2,11]$. For the case of the rot-rot formulation of the Maxwell equations, optimized Schwarz methods were developed in [1]. The systematic study of Schwarz methods for Maxwell's equations in their general formulation was started in $[9,10]$, and an entire hierarchy of families of optimized Schwarz methods was analyzed in [8], see also [5] for discontinuous Galerkin discretizations and large scale experiments. We present in this paper a first analysis of optimized Schwarz methods for Maxwell's equations with non-zero electric conductivity. We illustrate our analysis with numerical experiments.

\section{Schwarz Methods for Maxwell's Equations}

The time dependent Maxwell equations are

$$
-\varepsilon \frac{\partial \mathcal{E}}{\partial t}+\operatorname{curl} \mathcal{H}-\sigma \mathcal{E}=\boldsymbol{J}, \quad \mu \frac{\partial \mathcal{H}}{\partial t}+\operatorname{curl} \mathcal{E}=0,
$$

where $\mathcal{E}=\left(\mathcal{E}_{1}, \mathcal{E}_{2}, \mathcal{E}_{3}\right)^{T}$ and $\mathcal{H}=\left(\mathcal{H}_{1}, \mathcal{H}_{2}, \mathcal{H}_{3}\right)^{T}$ denote the electric and magnetic fields, respectively, $\varepsilon$ is the electric permittivity, $\mu$ is the magnetic permeability, $\sigma$ is the electric conductivity and $\mathcal{J}$ is the applied current density. We assume the applied current density to be divergence free, $\operatorname{div} \mathcal{J}=0$.

One can show, see for example [8] for the context of domain decomposition methods, that the time dependent Maxwell equations (1) are a system of hyperbolic partial differential equations. This hyperbolic system has for any interface two incoming and two outgoing characteristics. Imposing incoming characteristics is equivalent to imposing the impedance condition 


$$
\mathcal{B} \boldsymbol{n}(\mathcal{E}, \mathcal{H}):=\boldsymbol{n} \times \frac{\mathcal{E}}{Z}+\boldsymbol{n} \times(\mathcal{H} \times \boldsymbol{n})=\boldsymbol{s} .
$$

We consider in this paper the time-harmonic Maxwell equations,

$$
-i \omega \varepsilon \boldsymbol{E}+\operatorname{curl} \boldsymbol{H}-\sigma \boldsymbol{E}=\boldsymbol{J}, \quad i \omega \mu \boldsymbol{H}+\operatorname{curl} \boldsymbol{E}=\mathbf{0} .
$$

A family of Schwarz methods for (3) with a possibly non-overlapping decomposition of the domain $\Omega$ into $\Omega_{1}$ and $\Omega_{2}$, with interfaces $\Gamma_{12}:=\partial \Omega_{1} \cap \Omega_{2}$ and $\Gamma_{21}:=\partial \Omega_{2} \cap \Omega_{1}$, is given by

$$
\begin{array}{rlrl}
-i \omega \varepsilon \boldsymbol{E}^{1, n}+\operatorname{curl} \boldsymbol{H}^{1, n}-\sigma \boldsymbol{E}^{1, n} & =\boldsymbol{J} & & \text { in } \Omega_{1}, \\
i \omega \mu \boldsymbol{H}^{1, n}+\operatorname{curl} \boldsymbol{E}^{1, n} & =\mathbf{0} & & \text { in } \Omega_{1}, \\
\left(\mathcal{B}_{\boldsymbol{n}_{1}}+\mathcal{S}_{1} \mathcal{B}_{\boldsymbol{n}_{2}}\right)\left(\boldsymbol{E}^{1, n}, \boldsymbol{H}^{1, n}\right) & =\left(\mathcal{B}_{\boldsymbol{n}_{1}}+\mathcal{S}_{1} \mathcal{B} \boldsymbol{n}_{2}\right)\left(\boldsymbol{E}^{2, n-1}, \boldsymbol{H}^{2, n-1}\right) & \text { on } \Gamma_{12}, \\
-i \omega \varepsilon \boldsymbol{E}^{2, n}+\operatorname{curl} \boldsymbol{H}^{2, n}-\sigma \boldsymbol{E}^{2, n} & =\boldsymbol{J} & & \text { in } \Omega_{2}, \\
i \omega \mu \boldsymbol{H}^{2, n}+\operatorname{curl} \boldsymbol{E}^{2, n} & =\mathbf{0} & & \text { in } \Omega_{2}, \\
\left(\mathcal{B}_{\boldsymbol{n}_{2}}+\mathcal{S}_{2} \mathcal{B}_{\boldsymbol{n}_{1}}\right)\left(\boldsymbol{E}^{2, n}, \boldsymbol{H}^{2, n}\right) & =\left(\mathcal{B}_{\boldsymbol{n}_{2}}+\mathcal{S}_{2} \mathcal{B}_{\boldsymbol{n}_{1}}\right)\left(\boldsymbol{E}^{1, n-1}, \boldsymbol{H}^{1, n-1}\right) & \text { on } \Gamma_{21},
\end{array}
$$

where $\mathcal{S}_{j}, j=1,2$ are tangential, possibly pseudo-differential operators. Different choices of $\mathcal{S}_{j}, j=1,2$ lead to different parallel solvers for Maxwell's equations, see [8]. The classical Schwarz method is exchanging characteristic information at the interfaces between subdomains, which means $\mathcal{S}_{j}=0$, $j=1,2$. For the case of constant coefficients and the domain $\Omega=\mathbb{R}^{3}$, with the Silver-Müller radiation condition

$$
\lim _{r \rightarrow \infty} r(\boldsymbol{H} \times \boldsymbol{n}-\boldsymbol{E})=0
$$

and the two subdomains

$$
\Omega_{1}=(0, \infty) \times \mathbb{R}^{2}, \quad \Omega_{2}=(-\infty, L) \times \mathbb{R}^{2}, \quad L \geq 0,
$$

the following convergence result was obtained in [8] using Fourier analysis:

Theorem 1. For any $\left(\boldsymbol{E}^{1,0} ; \boldsymbol{H}^{1,0}\right) \in\left(L^{2}\left(\Omega_{1}\right)\right)^{6},\left(\boldsymbol{E}^{2,0} ; \boldsymbol{H}^{2,0}\right) \in\left(L^{2}\left(\Omega_{2}\right)\right)^{6}$, the classical algorithm with $\sigma>0$ converges in $\left(L^{2}\left(\Omega_{1}\right)\right)^{6} \times\left(L^{2}\left(\Omega_{2}\right)\right)^{6}$. The convergence factor for each Fourier mode $\boldsymbol{k}:=\left(k_{y}, k_{z}\right)$ with $|\boldsymbol{k}|^{2}:=k_{y}^{2}+k_{z}^{2}$ is

$$
\rho_{c l a}(\boldsymbol{k}, \tilde{\omega}, \sigma, Z, L)=\left|\frac{\sqrt{|\boldsymbol{k}|^{2}-\tilde{\omega}^{2}+i \tilde{\omega} \sigma Z}-i \tilde{\omega}}{\sqrt{|\boldsymbol{k}|^{2}-\tilde{\omega}^{2}+i \tilde{\omega} \sigma Z}+i \tilde{\omega}} e^{-\sqrt{|\boldsymbol{k}|^{2}-\tilde{\omega}^{2}+i \tilde{\omega} \sigma Z} L}\right|,
$$

where $\tilde{\omega}:=\omega \sqrt{\varepsilon \mu}$, and $Z:=\sqrt{\frac{\mu}{\varepsilon}}$.

This result shows that if $\sigma>0$, the method converges, also without overlap, $L=0$, which is unusual for classical Schwarz methods, but normal for optimized ones, for an explanation, see [6]. If however the electric conductivity $\sigma=0$, then for $|\boldsymbol{k}|^{2}=\tilde{\omega}^{2}$ the convergence factor equals 1, and the method is stagnating for this frequency, and thus by continuity slow for nearby frequencies. In addition, if there is no overlap, $L=0$, we have $\rho_{\text {cla }}(\boldsymbol{k})<1$ only for the propagative modes, $|\boldsymbol{k}|^{2}<\tilde{\omega}^{2}$, and $\rho_{\text {cla }}(\boldsymbol{k})=1$ for evanescent modes, i.e. when $|\boldsymbol{k}|^{2} \geq \tilde{\omega}^{2}$; the method is now stagnating for all evanescent modes. Hence for $\sigma=0$, better transmission conditions were developed in [8]. The analysis in [8] does however not apply if the electric conductivity $\sigma>0$. 


\section{Analysis for Non-Zero Electric Conductivity}

We present now an analysis of algorithm (4), (6) for the case where the electric conductivity is non-zero, $\sigma>0$, in the special case of the two dimensional transverse magnetic Maxwell equations. For these equations, the unknowns are independent of $z$, and we have $\boldsymbol{E}=\left(0,0, E_{z}\right)$ and $\boldsymbol{H}=\left(H_{x}, H_{y}, 0\right)$. The results are again based on Fourier transforms, here in the $y$ direction with Fourier variable $k$.

Theorem 2. For $\sigma>0$, if $\mathcal{S}_{j}, j=1,2$ have the constant Fourier symbol

$$
\sigma_{j}=\mathcal{F}\left(\mathcal{S}_{j}\right)=-\frac{s-i \tilde{\omega}}{s+i \tilde{\omega}}, \quad s \in \mathbb{C}
$$

then the optimized Schwarz method (4), (6) has the convergence factor

$$
\rho_{\sigma}(\tilde{\omega}, Z, \sigma, L, k, s)=\left|\left(\frac{\sqrt{k^{2}-\tilde{\omega}^{2}+i \tilde{\omega} \sigma Z}-s}{\sqrt{k^{2}-\tilde{\omega}^{2}+i \tilde{\omega} \sigma Z}+s}\right) e^{-\sqrt{k^{2}-\tilde{\omega}^{2}+i \tilde{\omega} \sigma Z} L}\right| .
$$

Proof. Taking a Fourier transform in the $y$ variable of (4) with $\boldsymbol{J}=0$, the so-called error equations, we get

$$
\partial_{x}\left(\begin{array}{c}
\hat{E}_{z}^{j, n} \\
\hat{H}_{y}^{j, n}
\end{array}\right)=\left(\begin{array}{cc}
0 & i \omega \mu \\
\frac{k^{2}-\omega^{2} \varepsilon \mu+i \omega \mu \sigma}{i \omega \mu} & 0
\end{array}\right)\left(\begin{array}{c}
\hat{E}_{z}^{j, n} \\
\hat{H}_{y}^{j, n}
\end{array}\right)=: M\left(\begin{array}{c}
\hat{E}_{z}^{j, n} \\
\hat{H}_{y}^{j, n}
\end{array}\right), j=1,2 .
$$

The eigenvalues of the matrix $M$, and their corresponding eigenvectors are

$$
\lambda_{j}= \pm \lambda= \pm \sqrt{k-\tilde{\omega}^{2}+i \tilde{\omega} \sigma Z}, \quad \boldsymbol{v}_{j}=\left(\begin{array}{c}
\mp \frac{i \omega \mu}{\lambda} \\
1
\end{array}\right), \quad j=1,2,
$$

and therefore the solutions of (9) are given by

$$
\left(\hat{E}_{z}^{1, n}, \hat{H}_{y}^{1, n}\right)=\alpha_{1}^{n} \boldsymbol{v}_{1} e^{\lambda x}+\alpha_{2}^{n} \boldsymbol{v}_{2} e^{-\lambda x},\left(\hat{E}_{z}^{2, n}, \hat{H}_{y}^{2, n}\right)=\beta_{1}^{n} \boldsymbol{v}_{1} e^{\lambda x}+\beta_{2}^{n} \boldsymbol{v}_{2} e^{-\lambda x}
$$

Using the Silver-Müller radiation condition (5), we have $\alpha_{2}^{n}=\beta_{1}^{n}=0$, and inserting the solutions into the interface conditions in (4), we get

$$
\alpha_{1}^{n}=A \beta_{2}^{n-1} e^{-\lambda L}, \quad \beta_{2}^{n}=A \alpha_{1}^{n-1} e^{-\lambda L}, \text { with } A:=\frac{\lambda-s}{\lambda+s},
$$

and the definition $\rho_{\sigma}(\tilde{\omega}, Z, \sigma, L, k, s):=\left|\frac{\alpha_{1}^{n}}{\alpha_{1}^{n-2}}\right|^{\frac{1}{2}}$ leads to the result (8).

In a numerical implementation, the range of frequencies is bounded, $k \in$ $K:=\left[k_{\min }, k_{\max }\right]$, where the minimum frequency $k_{\min }>0$ is a constant depending on the geometry, and the maximum numerical frequency that can be represented on a mesh is $k_{\max }=\frac{C}{h}$ where $C$ is a constant. From Theorem 2, we can immediately get a convergence result for the classical Schwarz method that uses characteristic transmission conditions. 
Corollary 1. For $\sigma>0$, in the case of the classical Schwarz method, $\sigma_{j}=0$, $j=1,2$, the asymptotic convergence factor for small mesh size $h$ is

$$
\bar{\rho}_{\sigma}:=\max _{k \in K}\left(\rho_{\sigma}\right)= \begin{cases}1-\frac{4}{3}\left(9 \omega^{4} \sigma^{2} \mu^{3} \varepsilon C_{L}^{6}\right)^{\frac{1}{8}} h^{\frac{3}{4}}+O\left(h^{\frac{5}{4}}\right), & L=C_{L} h, \\ 1-\frac{\omega^{2} \sigma \sqrt{\mu^{3} \varepsilon}}{C^{3}} h^{3}+O\left(h^{5}\right), & L=0 .\end{cases}
$$

Proof. The proof is obtained by inserting $s=i \tilde{\omega}$ into (8), and then expanding the maximum of $\rho_{\sigma}$ over $k \in K$ for $h$ small.

In order to obtain a more efficient algorithm, we choose $\sigma_{j}, j=1,2$ such that $\rho_{\sigma}$ is minimal over the range of frequencies $k \in K$. We look for $s$ of the form $s=p(1+i)$, such that $p$ is solution of the min-max problem

$$
\min _{p \geq 0}\left(\max _{k \in K} \rho_{\sigma}(\tilde{\omega}, Z, \sigma, L, k, p(1+i))\right) .
$$

Theorem 3. For $\sigma>0$, and the non-overlapping case, $L=0$, the solution of the minmax problem (12) is for $h$ sufficiently small given by

$$
p^{*}=\frac{(\omega \sigma \mu)^{\frac{1}{4}} \sqrt{C}}{2^{\frac{1}{4}} \sqrt{h}} \quad \text { and } \quad \rho_{\sigma}^{*}=1-\frac{2^{\frac{3}{4}}(\omega \sigma \mu)^{\frac{1}{4}} \sqrt{h}}{\sqrt{C}}+O(h) .
$$

Proof. We assume that $p \geq p_{c}:=\sqrt{\frac{3 \sigma \omega \mu}{2}}$, a hypothesis that can be removed with an additional analysis, which is too long however for this short paper. Using the change of variables $\xi(k):=\Re\left(\sqrt{k^{2}-\omega^{2} \epsilon \mu+i \sigma \omega \mu}\right)$ and $y:=\sigma \omega \mu$, the convergence factor simplifies to

$$
\rho_{\sigma}(\tilde{\omega}, Z, \sigma, 0, k, p(1+i))=\sqrt{\frac{4 \xi^{2}(\xi-p)^{2}+(y-2 \xi p)^{2}}{4 \xi^{2}(\xi+p)^{2}+(y+2 \xi p)^{2}}}=: R(\xi, y, p) .
$$

Since the mapping $k \mapsto \xi(k)$ is increasing in $k$ for $k \geq 0$, we have

$$
\min _{p \geq 0}\left(\max _{k_{\min } \leq k \leq k_{\max }} \rho_{\sigma}(\tilde{\omega}, Z, \sigma, 0, k, p(1+i))\right)=\min _{p \geq 0}\left(\max _{\xi_{0} \leq \xi \leq \xi_{\max }} R(\xi, y, p)\right),
$$

where $\xi_{0}=\xi\left(k_{\min }\right)$ and $\xi_{\max }=\xi\left(k_{\max }\right)$. We start by studying the variation of $R$ for fixed $p$; the polynomial

$$
P(\xi)=\left(\xi^{2}-\frac{1}{2} y\right)\left(8 \xi^{4}+16 \xi^{2}\left(y-p^{2}\right)+2 y^{2}\right)
$$

is the numerator of the partial derivative of $R$ with respect to $\xi$. $P$ has at most three positive roots, and $\xi_{2}=\sqrt{\frac{y}{2}}$ is always a root. We now show that for $p \geq p_{c}, \xi_{2}$ is a maximum and the other two roots of $P$ can not be maxima. The second partial derivative of $R$ with respect to $\xi$ evaluated at $\xi_{2}$ is 


$$
\frac{\partial^{2} R}{\partial \xi^{2}}\left(\xi_{2}, y, p\right)=-4 \sqrt{\frac{2}{y}} \frac{\left(2 p^{2}-3 y\right) p}{|\sqrt{2} p-\sqrt{y}|(\sqrt{2} p+\sqrt{y})^{3}} .
$$

Since for $p \geq p_{c}$ by assumption, $\frac{\partial^{2} R}{\partial \xi^{2}}\left(\xi_{2}, y, p\right) \leq 0, \xi_{2}$ is a local maximum. Since $R\left(\xi_{0}, y, p\right) \leq 1$ and $\lim _{\xi \rightarrow+\infty} R(\xi, y, p)=1$, the other two roots of $P$ can not be maxima if $p \geq p_{c}$. Therefore the maximum of $R$ is either at $\xi_{0}, \xi_{2}$ or $\xi_{\max }$. But we also find that $R\left(\xi_{0}, y, p\right) \leq R\left(\xi_{2}, y, p\right)$ for $p \geq p_{c}$, which excludes $\xi_{0}$ as a candidate for the maximum. Moreover, for $\xi_{\max }$ sufficiently large we have $R\left(\xi_{2}, y, p_{c}\right) \leq R\left(\xi_{\max }, y, p_{c}\right)$, and for $p$ large, we have $R\left(\xi_{2}, y, p\right) \geq R\left(\xi_{\max }, y, p\right)$. By continuity, there exists at least one $p^{*}$ such that $R\left(\xi_{2}, y, p^{*}\right)=R\left(\xi_{\max }, y, p^{*}\right)$. Moreover, the computation of

$$
\frac{\partial R}{\partial p}(\xi, y, p)=-\frac{1}{R(\xi, y, p)} \frac{\left(p^{2}-\frac{1}{8} \frac{4 \xi^{4}+y^{2}}{\xi^{2}}\right)}{\left(4 \xi^{4}+8 \xi^{3} p+8 \xi^{2} p^{2}+y^{2}+4 y \xi p\right)^{2}}
$$

shows that the function $p \mapsto R\left(\xi_{2}, y, p\right)$ is monotonically increasing, and $p \mapsto$ $R\left(\xi_{\max }, y, p\right)$ is monotonically decreasing for $\xi_{\max }$ sufficiently large. Hence $p^{*}$ is unique and therefore the unique solution of the min-max problem. As we have

$R\left(\xi_{2}, y, p^{*}\right)=R\left(\xi_{\max }, y, p^{*}\right) \Longrightarrow p^{*}=\frac{\sqrt[4]{2 y} \sqrt{\xi_{\max }\left(y+2 \xi_{\max }^{2}+\xi_{\max } \sqrt{2} \sqrt{y}\right)}}{2 \xi_{\max }}$,

by expanding $p^{*}$ for $h$ small, $\left(\xi_{\max }=\xi(C / h)\right)$, we get the desired result.

A numerical example of the convergence factor is shown in Figure 1. We can see that for $\sigma>0$, the classical Schwarz algorithm does not have convergence problems any more close to the resonance frequency. Increasing $\sigma$ further improves the performance, since the maximum of the convergence factor decreases. We also see that the optimization, which is based on equioscillation, leads to a uniformly small contraction factor when $\sigma>0$, whereas in the case $\sigma=0$ a small region close to the resonance frequency needs to be excluded in order to minimized the convergence factor for the remaining frequencies.



Fig. 1. Convergence factors as function of the frequency parameter $|\boldsymbol{k}|$ 


\section{Numerical Results}

We present now some numerical tests in order to illustrate the performance of the algorithms. The domain $\Omega$ is partitioned into several subdomains $\Omega_{j}$. In each subdomain, we use a discontinuous Galerkin method (DG), see [5].

We first test the propagation of a plane wave in a homogeneous medium. The domain is $\Omega=(0,1)^{2}$, and the parameters are constant in $\Omega$, with $\varepsilon=\mu=1, \sigma=5$ and $\omega=2 \pi$. We impose on the boundary an incident field $\boldsymbol{W}^{i n c}=\left(H_{x}^{i n c}, H_{y}^{i n c}, E_{z}^{i n c}\right)=\left(\frac{k_{y}}{\mu \omega}, \frac{-k_{x}}{\mu \omega}, 1\right) e^{-i \boldsymbol{k} \cdot \boldsymbol{x}}$ with $\boldsymbol{k}=\left(k_{x}, k_{y}\right)=$ $\left(\omega \sqrt{\varepsilon-i \frac{\sigma}{\omega}}, 0\right), \boldsymbol{x}=(x, y)$. The domain $\Omega$ is decomposed into two subdomains $\Omega_{1}=(0,1 / 2) \times(0,1)$ and $\Omega_{2}=(1 / 2,1) \times(0,1)$. For this test case, the DG method is used with a uniform polynomial approximation of order one, two and three, denoted by $D G-P 1, D G-P 2$ and $D G-P 3$. The performance of the algorithm is shown in Figure 2. These results are in good agreement with

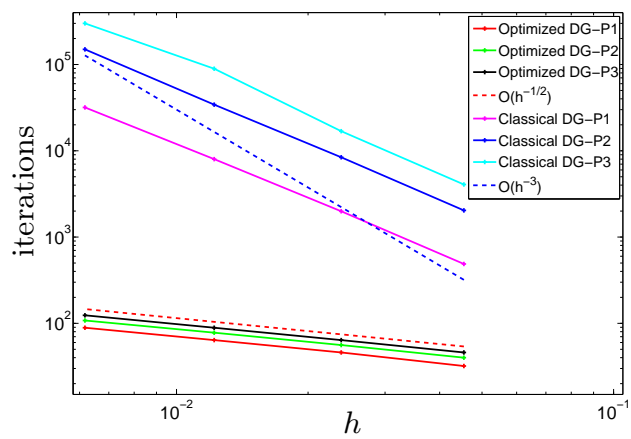

\begin{tabular}{ccccc}
\hline$h$ & $\frac{1}{10}$ & $\frac{1}{20}$ & $\frac{1}{40}$ & $\frac{1}{80}$ \\
\hline Optimized Schwarz & algorithm : \\
\hline$D G-P 1$ & 32 & 46 & 64 & 89 \\
\hline$D G-P 2$ & 40 & 56 & 78 & 108 \\
\hline$D G-P 3$ & 46 & 64 & 89 & 124 \\
\hline Classical & Schwarz & algorithm : \\
\hline$D G-P 1$ & 488 & 1990 & 8000 & 31821 \\
\hline$D G-P 2$ & 2038 & 8419 & 34262 & $15 \mathrm{E} 04$ \\
\hline$D G-P 3$ & 4066 & 16875 & 89486 & $30 \mathrm{E} 04$ \\
\hline
\end{tabular}

Fig. 2. Number of iterations against the mesh size $h$, to attain a relative residual reduction of $10^{-8}$ obtained with the classical and optimized Schwarz algorithm

the theoretical result in Theorem 3: the curves fit nicely the dependence on $h$ predicted, i.e they behave like $h^{-0.5}$. We also see the tremendous improvement of the optimized Schwarz method over the classical Schwarz method, which nevertheless performs a bit better than predicted in Corollary 1, the dependence on $h$ measured is $O\left(h^{-2}\right)$, instead of $O\left(h^{-3}\right)$; for an explanation, see $[7]$.

The second test problem is a simplified model of the propagation of an electromagnetic wave, emitted by a localized source, in the head tissues. The geometric configuration is given in Figure 3. The electromagnetic parameters of the material in the head tissues are: $\mu=1$ in the whole domain, $\varepsilon=43.85$, $\sigma=1.23 \cdot 120 \pi$ for the skin, $\varepsilon=15.56, \sigma=0.43 \cdot 120 \pi$ for the skull, $\varepsilon=67.20$, $\sigma=2.92 \cdot 120 \pi$ for the cerebrospinal fluid and $\varepsilon=43.55, \sigma=1.15 \cdot 120 \pi$ for the brain. The antenna is modeled by two perfectly conducting rods (with base section of $0.25^{2} \mathrm{~cm}^{2}$ ) and between these rods a current density $J_{z}$ is 


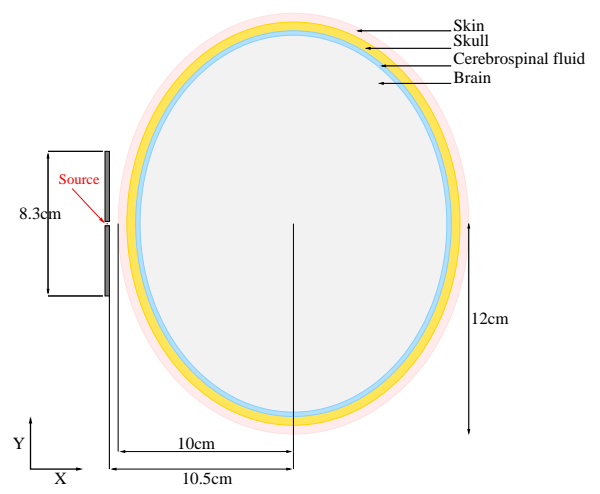

Fig. 3. Model of the different layers of a skull
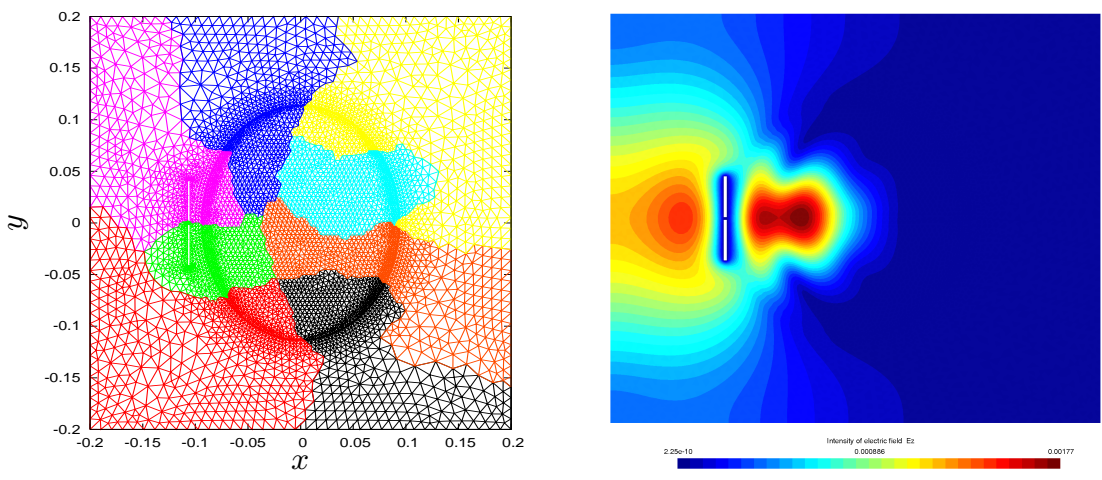

Fig. 4. Decomposition into subdomains and solution

applied. The computational domain is decomposed into several subdomains (a decomposition into eight subdomains is shown for example in Figure 4 on the left). We compare in this test the performance of the classical Schwarz and the new optimized Schwarz algorithm for a decomposition into two, four, eight and sixteen subdmains. In Table 1, we show the number of iterations needed for convergence, i.e to attain a relative residual of $10^{-8}$, depending on the number of subdomains. These results show that the optimized Schwarz algorithm converges much faster than the classical Schwarz algorithm. Here we used a Krylov method (BiCGStab) for the solution of the linear system, preconditioned with the classical and optimized Schwarz preconditioner.

\begin{tabular}{lrrrr}
\hline Number of subdomains & 2 & 4 & 8 & 16 \\
\hline \hline Classical Schwarz & 94 & 197 & 179 & 174 \\
\hline Optimized Schwarz & 69 & 92 & 82 & 85 \\
\hline
\end{tabular}

Table 1. Iteration number comparison for the cell phone antenna problem 


\section{Conclusion}

We analyzed an optimized Schwarz method for the two dimensional Maxwell equations with non-zero electric conductivity. The new method performs much better than the classical one, and our theoretical results are well confirmed by the numerical experiments presented, also for a non-trivial test case.

\section{References}

[1] A. Alonso-Rodriguez and L. Gerardo-Giorda. New nonoverlapping domain decomposition methods for the harmonic Maxwell system. SIAM J. Sci. Comput., 28(1):102-122, 2006.

[2] P. Chevalier and F. Nataf. An OO2 (Optimized Order 2) method for the Helmholtz and Maxwell equations. In 10th International Conference on Domain Decomposition Methods in Science and in Engineering, pages 400-407. AMS, 1997.

[3] B. Després. Décomposition de domaine et problème de Helmholtz. C.R. Acad. Sci. Paris, 1(6):313-316, 1990.

[4] B. Després, P. Joly, and J.E. Roberts. A domain decomposition method for the harmonic Maxwell equations. In Iterative methods in linear algebra, pages 475-484, Amsterdam, 1992. North-Holland.

[5] V. Dolean, M. El Bouajaji, M.J. Gander, S. Lanteri, and R. Perrussel. Domain decomposition methods for electromagnetic wave propagation problems in heterogeneous media and complex domains. In Domain Decomposition Methods in Science and Engineering XIX, 2010. submitted.

[6] V. Dolean and M.J. Gander. Why classical schwarz methods applied to hyperbolic systems can converge even without overlap. In Domain Decomposition Methods in Science and Engineering XVIII, pages 467476. Springer Verlag, 2007.

[7] V. Dolean and M.J. Gander. Can the discretization modify the performance of schwarz methods? In Domain Decomposition Methods in Science and Engineering XIX, 2010. submitted.

[8] V. Dolean, L. Gerardo-Giorda, and M.J. Gander. Optimized Schwarz methods for Maxwell equations. SIAM J. Scient. Comp., 31(3):2193$2213,2009$.

[9] V. Dolean, S. Lanteri, and R. Perrussel. A domain decomposition method for solving the three-dimensional time-harmonic Maxwell equations discretized by discontinuous Galerkin methods. J. Comput. Phys., $227(3): 2044-2072,2008$.

[10] V. Dolean, S. Lanteri, and R. Perrussel. Optimized Schwarz algorithms for solving time-harmonic Maxwell's equations discretized by a discontinuous Galerkin method. IEEE. Trans. Magn., 44(6):954-957, 2008.

[11] M.J. Gander, F. Magoulès, and F. Nataf. Optimized Schwarz methods without overlap for the Helmholtz equation. SIAM J. Sci. Comput., 24(1):38-60, 2002. 